\title{
Enfermedad de Caroli: revisión sistemática de la literatura
}

\section{Characterization of patients with Caroli’s disease: Systematic review}

\author{
Bryan Felipe Vacca-Carvajal, ${ }^{1 *}$ (1) José Mario Vásquez-Iles, ${ }^{1}$ María Gabriela Rangel-Pereira, ${ }^{1}$ Ledmar Jovanny Vargas-Rodríguez. ${ }^{2}$ (]
}

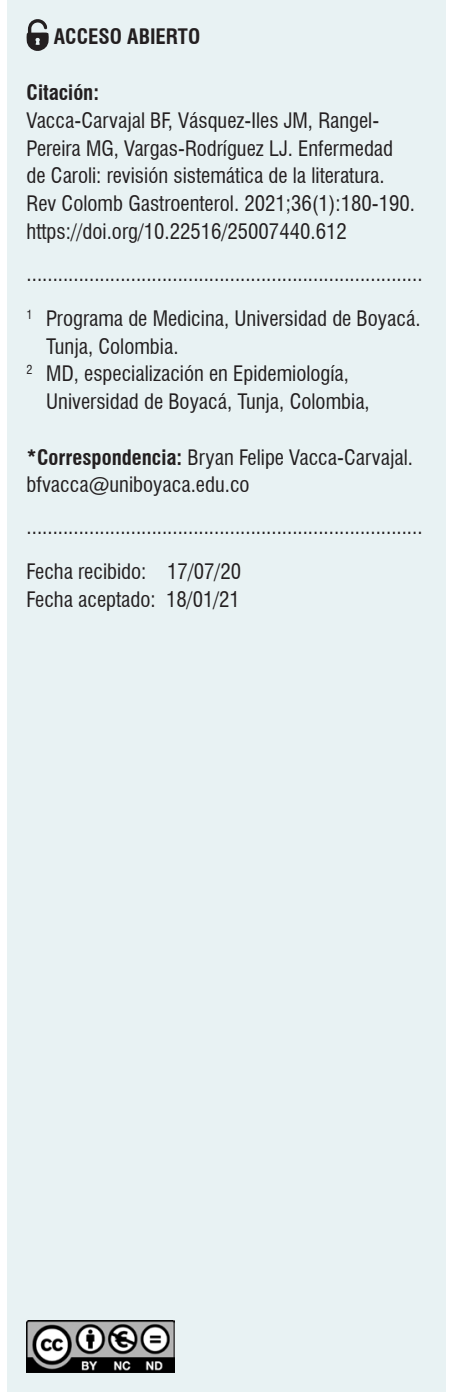

\begin{abstract}
Resumen
Introducción: la enfermedad de Caroli es un trastorno congénito poco usual, el cual cursa con dilatación segmentaria multifocal de los conductos biliares intrahepáticos. Fue descrita por Jacques Caroli en 1958 como una dilatación sacular, segmentada o fusiforme de los ductos biliares intrahepáticos. Objetivo: caracterizar a la población que ha padecido la enfermedad de Caroli. Metodología: Revisión sistemática de la literatura. Resultados: se analizaron 66 artículos, el grupo de edad más afectado es los menores de 10 años, en el que se evidenció una mayor prevalencia en varones, y se encontró asociado con otras comorbilidades como el riñón poliquístico (20\%). La manifestación más frecuente fue la hepatomegalia ( $44,7 \%)$, seguido de la fiebre $(42,4 \%)$ y el dolor abdominal en el hipocondrio derecho $(41,2 \%)$. Como método diagnóstico más utilizado se encontraba la resonancia magnética $(73,8 \%)$; dentro de los hallazgos predominó la dilatación intrahepática $(76,5 \%)$ y el manejo más empleado fue la antibioticoterapia para tratar las recurrencias por colangitis. Conclusión: la enfermedad de Caroli tiene una baja prevalencia, se presenta con mayor frecuencia en el continente americano, afecta principalmente a la primera década de la vida, tiene predilección por el sexo masculino y se caracteriza por una dilatación de los conductos intrahepáticos que pueden afectar a otros órganos como los riñones, lo que produce quistes renales.
\end{abstract}

\section{Palabras clave}

Enfermedad de Caroli, enfermedad quística del hígado, dilatación intrahepática, hipertensión portal, dilatación quística, enfermedades del recién nacido.

\begin{abstract}
Caroli's disease (CD) is a rare congenital disease, which presents with multifocal segmental intrahepatic bile duct dilatation. It was first described by Jacques Caroli in 1958 as a saccular or fusiform dilatation of the intrahepatic bile ducts. Objective: To characterize the population that has been diagnosed with Caroli's disease. Materials and methods: Systematic review. Results: 66 articles were analyzed. The age group most affected was children under 10 years old, where a higher prevalence was evidenced in males. This condition was associated with other comorbidities such as polycystic kidney in $20 \%$. The most frequent manifestation was hepatomegaly $(44.7 \%)$, followed by fever $(42.4 \%)$, and abdominal pain in the right upper quadrant $(41.2 \%)$. The most used diagnostic method was magnetic resonance imaging in $73.8 \%$ of the sample. The findings showed predominance of intra-hepatic dilatation in $76.5 \%$. The most widely used treatment was antibiotic therapy to treat recurrences due to cholangitis. Conclusion: Caroli's disease has an extremely low incidence and occurs more frequently in the American continent, affecting mainly patients in the first decade of life, with a predilection for the male sex. It is characterized by a dilatation of the intrahepatic ducts that can affect other organs such as the kidneys, causing renal cysts.
\end{abstract}

\section{Keywords}

Caroli's disease; Cystic liver disease; Intrahepatic dilatation; Portal hypertension; Hepatomegaly; Cystic dilation; Newborn diseases. 


\section{INTRODUCCIÓN}

La enfermedad de Caroli es una malformación congénita inusual de los conductos biliares intrahepáticos, que se caracterizan por presentar ectasia y dilatación de los mismos, por lo que se llega a alterar todo el tracto biliar (1). Cuando esta dilatación del árbol biliar cursa junto a la fibrosis hepática se denomina síndrome de Caroli (2).

Para esta entidad patológica existe una clasificación que fue creada por Alonso-Lej y modificada por Todani en 1977 (3, 4), la cual se muestra en la Tabla 1.

Tabla 1. Clasificación de Todani

\begin{tabular}{|clc|}
\hline Tipo & \multicolumn{1}{c}{ Descripción } & Frecuencia \\
\hline I & Dilatación segmentaria o fusiforme del colédoco & $80 \%-90 \%$ \\
\hline II & Divertículo sacular del colédoco extrahepático & $0 \%-2 \%$ \\
\hline III & $\begin{array}{l}\text { Dilatación de la porción intraduodenal del } \\
\text { colédoco (coledococele) }\end{array}$ & $1 \%$ \\
\hline IV & $\begin{array}{l}\text { Múltiples dilataciones quísticas de la vía biliar } \\
\text { intra- y extrahepática (IVa) o únicamente } \\
\text { extrahepática (IVb) }\end{array}$ & $10 \%-15 \%$ \\
\hline V & $\begin{array}{l}\text { Afectación difusa quística de la vía biliar } \\
\text { intrahepática (enfermedad de Caroli) }\end{array}$ & $20 \%$ \\
\hline
\end{tabular}

La enfermedad de Caroli tiene un componente genético asociado con otras enfermedades hepáticas y renales, y es de mayor interés la relación con las enfermedades renales poliquísticas. Su origen genético está involucrado con la mutación del gen PKHD 1, que además es responsable de la proteína fibrocistina; asimismo, se conoce que su expresión se ve principalmente en el riñón y de manera secundaria en el hígado y páncreas $(1,5)$.

La mayor parte de los casos cursa con heterocigosidad compuesta para este gen, lo que explica que ambos alelos tienen 2 mutaciones diversas, las cuales hacen que el gen sea defectuoso y conlleve a una proteína de fibrocistina anormal. Esto refleja la diversidad de la base de mutación que experimenta el gen PKHD1 $(1,5)$.

Además de esto, su presentación es inusual: se presenta en menos de 1 por cada 1000000 de habitantes (5). A pesar de toda la literatura conocida sobre esta patología, no ha sido posible establecer de manera clara y concisa los factores de riesgo que se encuentran directamente implicados en la aparición de la enfermedad $(1,5)$.

El cuadro clínico puede ser variado; en algunos pacientes es posible que se manifieste como un dolor abdominal, colangitis con su consecuente fiebre, ictericia, pancreatitis, pérdida de peso y astenia. Su presentación clínica puede llegar hasta una enfermedad hepática en terminal.
Entre los métodos diagnósticos se encuentran la biopsia hepática, la colangiorresonancia magnética nuclear y la ecografía abdominal $(2,5)$.

La enfermedad de Caroli es conocida por ser una patología agresiva de los conductos intrahepáticos, por lo que es frecuente encontrar secuelas en los pacientes que padecen esta patología (6-8). El desenlace de la enfermedad de Caroli puede ser exitoso o un fracaso en el abordaje terapéutico, a tal punto que el último recurso para manejar a un paciente es someterlo a un trasplante hepático, con el fin de poder evitar la mortalidad temprana; no obstante, en algunos casos este método es insuficiente o no aplicado y terminan falleciendo inevitablemente (9-11).

El objetivo de este estudio es realizar una caracterización de la presentación clínica, el diagnóstico y manejo instaurado en la enfermedad, con base en la revisión sistemática de la literatura.

\section{MATERIALES Y MÉTODOS}

\section{Tipo de estudio}

Se realizó una búsqueda sistemática de la literatura con base en la declaración PRISMA 20 y en el Manual Cochrane de revisiones sistemáticas de intervenciones, versión 5.1.022.

\section{Población}

Se incluyó toda la población que presentó diagnóstico de enfermedad de Caroli, que hubiesen sido tratados y publicados entre 1979 y 2019 ; y se analizaron las características sociodemográficas, clínicas y terapéuticas, y el pronóstico para determinar los factores que estén asociados con la presencia de la enfermedad.

\section{Criterios de inclusión}

Se incluyeron artículos de reportes, series de casos, estudios transversales, casos y controles, cohortes y ensayos clínicos en los que se contaran con datos sociodemográficos, clínicos, imagenológicos y de tratamiento relacionados con la enfermedad de Caroli (enfermedad quística del hígado).

\section{Criterios de exclusión}

Se excluyeron las revisiones de temas, las cartas al editor $\mathrm{y}$ los estudios que no fueran realizados en seres humanos.

\section{Variables}

Las variables que se incluyeron fueron sexo $(M / F)$, país, año de publicación, antecedentes (patológicos [agenesia renal, 
artritis reumatoidea, colangitis, desnutrición crónica, dispepsia, espongiosis medular renal, fístula biliar persistente, hepatitis, ictericia neonatal, oligohidramnios, pielonefritis, várices esofágicas, várices gástricas, enfermedad poliquística renal y hepática, antecedente familiar de enfermedad renal, cirrosis, enfermedad renal crónica, hipertensión portal, riñón poliquístico], quirúrgicos [coledocoyeyunostomía en Y de Roux, ligadura de várices esofágicas, colecistectomía laparoscópica, trasplante renal, laparotomía]), manifestaciones clínicas (prurito, heces acolias, pérdida de apetito, pérdida de peso, distensión abdominal, epigastralgia, ictericia, esplenomegalia, dolor abdominal generalizado, náuseas/vómitos, dolor abdominal en el hipocondrio derecho, fiebre, hepatomegalia), métodos diagnósticos (angiografía abdominal, colangiografía intraoperatoria, coledocografía, colangiografía percutánea transhepática, gammagrafía de vías biliares, endoscopia de las vías digestivas altas [EVDA], gammagrafía, urografía, radiografía, colangiopancreatografía retrógrada endoscópica [CPRE], tomografía, ecografía, resonancia), características de la patología (hallazgos y lóbulo afectado), manejo y secuelas.

\section{Estrategia de búsqueda de la literatura}

La búsqueda estuvo a cargo de 3 investigadores, los cuales la realizaron entre el 10 y el 28 de septiembre del 2019. Las bases de datos utilizadas fueron Pubmed/Medline, Scopus, Embase, Scielo y Science Direct, en las que se realizó la búsqueda usando las siguientes palabras claves (DeCS $[\mathrm{MeSH}])$ : enfermedad de Caroli (Caroli Disease), dilatación intrahepática (intrahepatic dilation) y enfermedad quística del hígado (cystic liver disease).

\section{Restricciones en la búsqueda}

Se limitó la búsqueda a la literatura relacionada con humanos, publicados en los idiomas inglés y español.

\section{Extracción de datos}

La extracción de los datos estuvo a cargo de 3 investigadores pertenecientes al proyecto, quienes como estrategia de búsqueda realizaron la identificación y la detección de la literatura a estudiar. Luego, se seleccionaron los artículos según el titulo para elegir aquellos que fueran acordes al objetivo planteado. Posteriormente, se evaluaron los resúmenes de las investigaciones seleccionadas y, finalmente, se revisaron los artículos completos para ser elegidos e incluidos en el presente estudio.

Finalmente, se realizó una base de datos que fue registrada en Excel versión 2013 y se analizó con el paquete estadístico SPSS versión 22; el análisis univariado se realizó por medio de estadística descriptiva, determinando frecuencias absolutas y relativas en las variables cualitativas, mientras que en las variables cuantitativas se estimaron medidas de tendencia central y de dispersión.

\section{Sesgos}

En este diseño de estudio hubo varios sesgos a controlar. El primero fue la mala selección de los participantes, y para evitarlo se desarrollaron criterios de inclusión y exclusión. El segundo sesgo fue el de la medición, y para corregirlo se utilizó una ficha de recolección de datos que fue aplicado por 3 investigadores. El tercer sesgo es el de los vacíos en el registro de la información, en el que los datos son obtenidos de los registros de reportes de caso y series de casos publicados con la posibilidad de que la información recolectada esté incompleta.

\section{RESULTADOS}

\section{Selección de estudios}

Mediante la estrategia de búsqueda planteada se encontraron 854 artículos en las bases de datos; luego, al aplicar los límites de búsqueda se eliminaron 517 estudios y quedaron 340 artículos. Posteriormente, se hizo la revisión del título y resumen, con lo que se eliminaron 245 publicaciones, para un total de 95 artículos. Después de la revisión completa de los manuscritos se consideró que 17 no cumplían con los criterios para dar respuesta al estudio. De los 78 artículos restantes, se eliminaron 12 , que se encontraban repetidos, con lo cual se obtuvo un total de 66 artículos publicados que correspondían a reportes y series de casos; sin embargo, no se hallaron artículos de estudios transversales, casos y controles, cohortes y ensayos clínicos. Por tanto, el presente estudio tiene como limitación la dificultad para ser una inferencia causal.

El proceso de selección de dichos estudios se muestra a través del diagrama de flujo (Figura 1), de acuerdo con la declaración de PRISMA.

\section{Características sociodemográficas de los pacientes}

$\mathrm{Al}$ momento de recolectar y analizar los datos acerca de la edad de presentación más frecuente (Tabla 2), se pudo encontrar que la mayor prevalencia de la enfermedad está en un rango aproximado de 0 a 40 años con 63 pacientes reportados; además, se pudieron hallar picos característicos en las edades de 0-10 años (32,9\%), 20-30 años (22,4\%) y, por último, 30-40 años $(14,1 \%)(9,12)$.

El sexo con mayor presentación de esta enfermedad es el masculino (48 casos), con un porcentaje de 56,5 \% (13-18). 


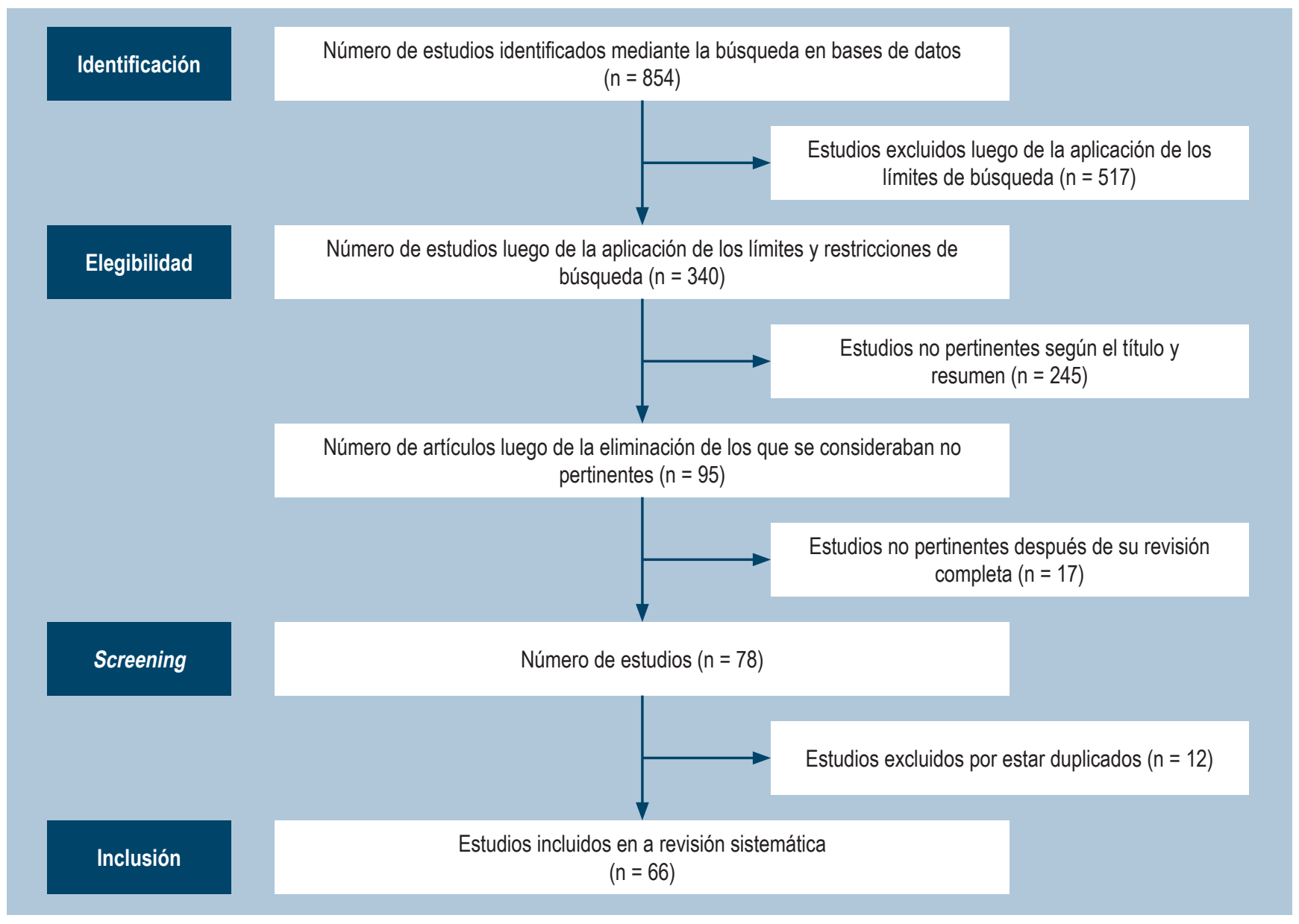

Figura 1. Flujograma PRISMA para la selección de estudios.

Además, se identificó que el país que reportó más casos de esta patología resultó ser Estados Unidos con un 17,6 \% $(12,19,20)$. Los antecedentes patológicos que con mayor frecuencia se han asociado con el desarrollo de esta patología, en orden descendente de presentación, son el riñón poliquístico ( 17 casos: $20 \%$ ), hipertensión portal ( 16 casos: $18,8 \%)$ y ERC ( 8 casos: $9,4 \%)(19,21,22)$. Otra variable de relevancia es los antecedentes quirúrgicos, en los que no se evidenciaron datos relevantes, puesto que se obtuvo una similitud de resultados y no se evidenció un factor con mayor impacto en los resultados. La colecistectomía laparoscópica, el trasplante renal y la laparotomía se practicaron en un 3,6\% y la coledocoyeyunostomía en Y de Roux con la ligadura de várices esofágicas, en un 1,2\% (23-25).

\section{Características clínicas}

Se realizó el análisis de la serie de casos clínicos revisados por los investigadores quienes encontraron que las manifestaciones más frecuentes en la enfermedad de
Caroli son la hepatomegalia, en 38 pacientes $(44,7 \%)$ (26-30); seguida del dolor en el hipocondrio derecho, en 35 pacientes ( $41,2 \%)$; la esplenomegalia, en 20 pacientes $(23,4 \%)$; y, por último, la ictericia, en 19 casos $(22,4 \%)$. El $42,4 \%$ de los pacientes presentó fiebre, que es una manifestación de la colangitis como complicación de esta patología (Tabla 3 ) (31-36).

De acuerdo con los casos clínicos seleccionados por el grupo de investigación, se observó que las 3 herramientas diagnósticas más utilizadas al momento de generar un posible diagnóstico fueron, en primer lugar, la resonancia magnética (RMN), en 62 pacientes $(73,8 \%)$; seguida por la ecografía, en 35 pacientes $(41,6 \%)$; la tomografía, con un porcentaje de uso de $38,1 \%$; y la CPRE, en un $28,57 \%$. A pesar de que la ecografía es el segundo método diagnóstico más utilizado, sirve como tamizaje y se requiere confirmación mediante colangiorresonancia (37-41).

La enfermedad de Caroli es un trastorno congénito poco frecuente, por lo que los principales estudios realizados en los diferentes pacientes mostraron hallazgos importantes 
(Tabla 4), y uno de los principales fue la dilatación intrahepática, que se presentó en 65 pacientes con un porcentaje de $76,5 \%$; por ende, se presentó el colédoco dilatado en 37 pacientes, con un porcentaje de $43,5 \%$; y el siguiente hallazgo fue una colelitiasis en 26 pacientes, con un porcentaje de $30,6 \%$, que provocó quistes renales en 17 pacientes, equivalentes a $20,0 \%(42-45)$.
De acuerdo con la fisiopatología de la enfermedad de Caroli, esta puede situarse de manera unilateral y bilateral; por consiguiente, en el presente análisis es posible denotar una inclinación de esta patología a presentarse de manera bilateral en los lóbulos hepáticos en 48 casos (56,5\%); en segundo lugar, en el lóbulo derecho con 22 casos $(25,9 \%)$ y, finalmente, en el lóbulo izquierdo con 15 casos $(17,6 \%)(6,10,46-48)$.

Tabla 2. Características sociodemográficas

\begin{tabular}{|c|c|c|c|c|c|}
\hline Variable & Prevalencia & $n$ & $\%$ & \multicolumn{2}{|c|}{ IC $95 \%$} \\
\hline \multirow[t]{8}{*}{ Edad } & $0-10$ & 28 & 32,9 & 22,95 & 42,93 \\
\hline & $10-20$ & 4 & 4,7 & 0,2043 & 9,207 \\
\hline & $20-30$ & 19 & 22,4 & 13,5 & 31,21 \\
\hline & $30-40$ & 12 & 14,1 & 6,716 & 21,52 \\
\hline & $40-50$ & 8 & 9,4 & 3,205 & 15,62 \\
\hline & $50-60$ & 7 & 8,2 & 2,392 & 14,08 \\
\hline & $60-70$ & 6 & 7,1 & 1,614 & 12,5 \\
\hline & $>70$ & 1 & 1,2 & 0,0 & 3,469 \\
\hline \multirow[t]{2}{*}{ Sexo } & Femenino & 37 & 43,5 & 32,99 & 54,07 \\
\hline & Masculino & 48 & 56,5 & 45,93 & 67,01 \\
\hline \multirow[t]{19}{*}{ Antecedentes patológicos } & Agenesia renal & 1 & 1,2 & 0,0 & 3,469 \\
\hline & Artritis reumatoidea & 1 & 1,2 & 0,0 & 3,469 \\
\hline & Colangitis & 1 & 1,2 & 0,0 & 3,469 \\
\hline & Desnutrición crónica & 1 & 1,2 & 0,0 & 3,469 \\
\hline & Dispepsia & 1 & 1,2 & 0,0 & 3,469 \\
\hline & Espongiosis medular renal & 1 & 1,2 & 0,0 & 3,469 \\
\hline & Fístula biliar persistente & 1 & 1,2 & 0,0 & 3,469 \\
\hline & Hepatitis & 1 & 1,2 & 0,0 & 3,469 \\
\hline & Ictericia neonatal & 1 & 1,2 & 0,0 & 3,469 \\
\hline & Oligohidramnios & 1 & 1,2 & 0,0 & 3,469 \\
\hline & Pielonefritis & 1 & 1,2 & 0,0 & 3,469 \\
\hline & Várices esofágicas & 1 & 1,2 & 0,0 & 3,469 \\
\hline & Várices gástricas & 1 & 1,2 & 0,0 & 3,469 \\
\hline & Enfermedad poliquística renal y hepática & 2 & 2,4 & 0,0 & 5,575 \\
\hline & Antecedente familiar de enfermedad renal & 3 & 3,5 & 0,0 & 7,452 \\
\hline & Cirrosis & 5 & 5,9 & 0,8806 & 10,88 \\
\hline & ERC & 8 & 9,4 & 3,205 & 15,62 \\
\hline & Riñón poliquístico & 17 & 20 & 11,5 & 28,5 \\
\hline & Coledocoyeyunostomía en Y de Roux & 1 & 1,2 & 0,0 & 3,469 \\
\hline \multirow[t]{4}{*}{ Antecedentes quirúrgicos } & Ligadura de várices esofágicas & 1 & 1,2 & 0,0 & 3,469 \\
\hline & colecistectomía laparoscópica & 3 & 3,6 & 0,0 & 7,452 \\
\hline & Trasplante renal & 3 & 3,6 & 0,0 & 7,452 \\
\hline & Laparotomía & 3 & 3,6 & 0,0 & 7,452 \\
\hline
\end{tabular}

ERC: enfermedad renal crónica; IC: intervalo de confianza. 


\section{Características terapéuticas y pronóstico}

Dentro de los posibles abordajes terapéuticos que pueden ser utilizados para la enfermedad de Caroli (Tabla 4) se encontró una inclinación hacia la utilización de la antibioticoterapia, con 40 casos (47,1\%); la CPRE, con 35 casos (41,2\%); el ácido ursodesoxicólico, con 9 casos (10,6\%); y 14 casos de trasplante $(16,5 \%)(12,15,49-56)$.

La complicación más frecuente que se evidenció fue la colangitis recurrente ( 6 casos), lo que representa el 7,1 \% del total de casos (6-8). En esta investigación hubo 8 casos de mortalidad, lo que equivale al 9,4\% del conjunto de casos (9-11).

\section{DISCUSIÓN}

Antiguamente, el acceso a los estudios imagenológicos era limitado y de difícil alcance y las ayudas imagenológicas no eran utilizadas de manera frecuente en la atención de estos pacientes; debido a esto, se pudo concluir que esta enfermedad muy probablemente fue subdiagnosticada (57). Por consiguiente, uno de los objetivos de esta revisión fue la caracterización de los pacientes que presentan enfermedad de Caroli, puesto que es un trastorno infrecuente que comprende una cantidad de anormalidades, entre las cuales se encuentran la dilatación sacular intrahepática, dilatación del colédoco, formación de cálculos y colangitis (19).

Por una parte, los reportes de casos sobre esta enfermedad son escasos; no obstante, resultó evidente que la edad de presentación con más frecuencia fue de 0-10 años de edad (32,9\%), seguida de los de 20-30 años $(22,4 \%)$ y de los de 30-40 años (14,1\%). Por otra parte, si se toma el rango de edad de 0 a 40 años, es posible encontrar una prevalencia de 63 pacientes; por tanto, se deduce que la enfermedad

Tabla 3. Características clínicas

\begin{tabular}{|c|c|c|c|c|c|}
\hline Variable & Prevalencia & $\mathbf{n}$ & $\%$ & \multicolumn{2}{|c|}{ IC $95 \%$} \\
\hline \multirow[t]{13}{*}{ Manifestaciones clínicas } & Prurito & 3 & 3,5 & 0,0 & 7,452 \\
\hline & Heces acólicas & 4 & 4,7 & 0,2043 & 9,207 \\
\hline & Pérdida de apetito & 8 & 9,4 & 3,205 & 15,62 \\
\hline & Pérdida de peso & 8 & 9,4 & 3,205 & 15,62 \\
\hline & Distensión abdominal & 13 & 15,3 & 7,643 & 22,95 \\
\hline & Epigastralgia & 17 & 20 & 11,5 & 28,5 \\
\hline & Ictericia & 19 & 22,4 & 13,5 & 31,21 \\
\hline & Esplenomegalia & 20 & 23,52 & 14,51 & 32,55 \\
\hline & Dolor abdominal generalizado & 29 & 34,1 & 24,04 & 44,2 \\
\hline & Náuseas/vómitos & 32 & 37,6 & 27,35 & 47,95 \\
\hline & Dolor abdominal del hipocondrio derecho & 35 & 41,2 & 30,71 & 51,64 \\
\hline & Fiebre & 36 & 42,4 & 31,85 & 52,86 \\
\hline & Hepatomegalia & 38 & 44,7 & 34,14 & 55,27 \\
\hline \multirow[t]{13}{*}{ Estudios realizados } & Angiografía abdominal & 2 & 2,38 & 0,0 & 5,575 \\
\hline & Colangiografías intraoperatorias & 2 & 2,38 & 0,0 & 5,575 \\
\hline & Coledocografía & 2 & 2,38 & 0,0 & 5,575 \\
\hline & Colangiografía percutánea transhepática & 3 & 3,57 & 0,0 & 7,452 \\
\hline & Gammagrafía de vías biliares & 4 & 4,76 & 0,2043 & 9,207 \\
\hline & EVDA & 5 & 5,95 & 0,8806 & 10,88 \\
\hline & Gammagrafía & 6 & 7,14 & 1,614 & 12,5 \\
\hline & Urografía & 7 & 8,33 & 2,392 & 14,08 \\
\hline & Radiografía & 8 & 9,52 & 3,205 & 15,62 \\
\hline & CPRE & 24 & 28,57 & 18,67 & 37,8 \\
\hline & Tomografía & 32 & 38,1 & 27,35 & 47,95 \\
\hline & Ecografía & 35 & 41,67 & 30,71 & 51,64 \\
\hline & Resonancia & 62 & 73,81 & 63,5 & 82,39 \\
\hline
\end{tabular}


Tabla 4. Hallazgos y características terapéuticas

\begin{tabular}{|c|c|c|c|c|c|}
\hline Variable & Prevalencia & $n$ & $\%$ & \multicolumn{2}{|c|}{ IC $95 \%$} \\
\hline \multirow[t]{7}{*}{ Hallazgos patognomónicos } & Tumor hepático & 7 & 8,2 & 2,392 & 14,08 \\
\hline & Dilatación de túbulos renales & 8 & 9,4 & 3,205 & 15,62 \\
\hline & Dilatación extrahepática & 10 & 11,8 & 4,916 & 18,61 \\
\hline & Quistes renales & 17 & 20 & 11,5 & 28,5 \\
\hline & Colelitiasis & 26 & 30,6 & 20,79 & 40,38 \\
\hline & Colédoco dilatado & 37 & 43,5 & 32,99 & 54,07 \\
\hline & Dilatación intrahepática & 65 & 76,5 & 67,45 & 85,49 \\
\hline \multirow[t]{3}{*}{ Lóbulo afectado } & Izquierdo & 15 & 17,6 & 9,543 & 25,75 \\
\hline & Derecho & 22 & 25,9 & 16,57 & 35,19 \\
\hline & Bilateral & 48 & 56,5 & 45,93 & 67,01 \\
\hline \multirow[t]{10}{*}{ Características terapéuticas } & Nefrectomía & 2 & 2,4 & 0,0 & 5,575 \\
\hline & Reconstrucción biliar & 3 & 3,5 & 0,0 & 7,452 \\
\hline & Inmunomoduladores (ciclosporina, tacrolimus, OKT3) & 4 & 4,7 & 0,2043 & 9,207 \\
\hline & Corticoides & 6 & 7,1 & 1,614 & 12,5 \\
\hline & Colecistectomía & 6 & 7,1 & 1,614 & 12,5 \\
\hline & Resección hepática segmentaria & 7 & 8,2 & 2,392 & 14,08 \\
\hline & Ácido ursodesoxicólico & 9 & 10,6 & 4,048 & 17,13 \\
\hline & Trasplante & 14 & 16,5 & 8,586 & 24,36 \\
\hline & Esfinterotomía & 35 & 41,2 & 30,71 & 51,64 \\
\hline & Antibioticoterapia (fluoroquinolonas, betalactámicos) & 40 & 47,1 & 36,45 & 57,67 \\
\hline \multirow[t]{5}{*}{ Secuelas } & Pielocaliectasia & 1 & 1,2 & 0,0 & 3,469 \\
\hline & Psoriasis & 1 & 1,2 & 0,0 & 3,469 \\
\hline & Esplenomegalia & 2 & 2,4 & 0,0 & 5,575 \\
\hline & Lesión hepática irreversible & 2 & 2,4 & 0,0 & 5,575 \\
\hline & Colangitis recurrente & 6 & 7,1 & 1,614 & 12,5 \\
\hline Mortalidad & & 8 & 9,4 & 3,205 & 15,62 \\
\hline
\end{tabular}

OKT3: muromonab-CD3.

de Caroli se presenta más en la población juvenil y adultez media, como lo afirman Saracíbar (58) y Shenoy (59).

Se dedujo que el sexo con mayor predisposición para esta patología es el masculino $(56,5 \%)$, a pesar de que existe un estudio que indica que el sexo femenino es el que se ve más afectado, con una relación hombre-mujer de 1:1,8 (1).

Un antecedente que sirve como factor predisponente, el cual tuvo mayor repercusión, fue el riñón poliquístico, con un $20 \%$; este hecho avala lo reportado por otros autores, quienes también afirman que el riñón poliquístico es un antecedente que se presenta con frecuencia en estos pacientes $(49,59-63)$.

Las manifestaciones clínicas de esta patología son diversas y pueden confundirse con otras patologías de tipo abdominal; no obstante, se encontró una inclinación hacia 3 manifestaciones principales: la hepatomegalia $(44,7 \%)$; fiebre $(42,4 \%)$ y dolor en el hipocondrio derecho $(41,2 \%)$, hallazgos similares a lo descrito por Carrera (60) y Ahmed (59). Por tanto, es indispensable el uso de ayudas diagnósticas con el fin de identificar correctamente la presencia de esta patología (64).

Se logró observar que la mayoría de los artículos en la década de los 90 refería que el mejor método diagnóstico para dicha patología era la ecografía y la tomografía, basado en lo que menciona Sood (13), Kaiser (19) y Sans (65). Aunque esto variaba, ya que muchos también mencionaban que la CPRE era el método más efectivo, porque mostraba una precisa anatomía del sistema biliar (16). 
Se puede inferir que el método diagnóstico más utilizado actualmente, según los reportes de casos revisados por los investigadores, fue la resonancia magnética $(73,81 \%)$, por su alto valor no invasivo y su excelente capacidad para mostrar el sistema biliar y la posible asociación con hipertensión portal y colangiocarcinoma $(62,64,66)$.

En cuanto a los hallazgos que se pudieron determinar mediante las ayudas diagnósticas, fue posible evidenciar dilatación intrahepática en el 76,5\% de los casos; asimismo, la literatura respalda esta postura, puesto que la dilatación intrahepática es un pilar fundamental en la enfermedad de Caroli $(12,67)$.

En definitiva, se pudo deducir que la presentación de la patología tuvo predilección por ambos lóbulos hepáticos, opuesto a lo que afirman Murcia (68) y Sinha (69), quienes mencionan que la afectación principalmente es de carácter unilateral con tendencia al lóbulo izquierdo.

Actualmente no existe un tratamiento específico para la enfermad Caroli, puesto que es una patología de baja prevalencia, de la cual no se conocen sus mecanismos fisiopatológicos; por tanto, el manejo se basa en el control de las complicaciones que se desarrollan en el curso de la enfermedad, como la colangitis, la cual se maneja con la administración de antibióticos; aunque cabe aclarar que el uso de estos no previene las recurrencias. Otra complicación que se puede presentar son los quistes renales y la insuficiencia renal crónica, la cual será tratada por los nefrólogos y depende del estadio en el que se encuentre (70). Finalmente, se puede presentar cirrosis hepática en los estadios avanzados de la enfermedad, en la que se pueden usar otras medidas terapéuticas como la lobectomía y el trasplante hepático $(71,72)$. En relación con lo anterior, se puede deducir que el abordaje médico oportuno es fundamental para el pronóstico de vida del paciente, con el fin de evitar al máximo la mortalidad; en lo que respecta al presente estudio, se hallaron 8 casos de muerte por enfermedad de Caroli, lo cual representa un 9,4\% de los casos $(57,71)$.

Las limitaciones a tener en cuenta en la presente investigación se deben al bajo número de estudios de investigación clínica, lo cual conllevó únicamente a la inclusión de reportes y series de caso, que aunque permiten realizar una adecuada caracterización de estos pacientes a nivel mundial, no permite establecer inferencia causal de esta patología; sin embargo, estimula a la realización de nuevas investigaciones de mayor jerarquía con el fin de conocer los factores de riesgo, asociaciones y medidas terapéuticas novedosas.

Finalmente, se pudo concluir que esta patología es de baja prevalencia y se presenta con mayor frecuencia en los países desarrollados, liderado por Estados Unidos, debido a que posee diversos estudios epidemiológicos, por lo que cuenta con una mayor incidencia de la enfermedad; contrario a lo que ocurre en los países subdesarrollados, donde no se encuentra una amplia variedad de estudios epidemiológicos que demuestren la presencia de esta enfermedad. Además, se determinó que la afectación se produce principalmente en la primera década de la vida; sin embargo, como se pudo observar en el presente estudio, la enfermedad de Caroli puede presentarse en cualquier etapa de la vida, con un predominio hacia el sexo masculino. Adicionalmente, se confirmó la prevalencia de la dilatación de los conductos intrahepáticos en esta enfermedad y que puede afectar a otros órganos adyacentes como los riñones, por lo que se producen quistes renales.

\section{Conflictos de interés}

Ninguno.

\section{Fuentes de financiación}

Ninguna.

\section{REFERENCIAS}

1. Cabral Correia P, Morgado B. Caroli's Disease as a Cause of Chronic Epigastric Abdominal Pain: Two Case Reports and a Brief Review of the Literature. Cureus. 2017;9(9):e1701.

https://doi.org/10.7759/cureus.1701

2. Fahrner R, Dennler SGC, Dondorf F, Ardelt M, Rauchfuss F, Settmacher U. Liver resection and transplantation in Caroli disease and syndrome. J Visc Surg. 2019;156(2):91-95. https://doi.org/10.1016/j.jviscsurg.2018.06.001

3. Uribarrena Amezaga R, Raventós N, Fuentes J, Elías J, Tejedo V, Uribarrena Echebarría R. Diagnóstico y trata- miento de los quistes de colédoco. Presentación de 10 nuevos casos. Rev Esp Enferm Dig. 2008;100(2):71-5. https://doi.org/10.4321/s1130-01082008000200002

4. Tello de Meneses Salazar A, Riggen Martínez L, Orozco Chávez EG. Quiste de colédoco tipo I: Diagnóstico por imagen y anastomosis hepático-duodenal como medida terapéutica. Bol. Med. Hosp. Infant. Mex. 2013;70(6):482-7.

5. Moslim MA, Gunasekaran G, Vogt D, Cruise M, MorrisStiff G. Surgical Management of Caroli’s Disease: Single Center Experience and Review of the Literature. J 
Gastrointest Surg. 2015;19(11):2019-27.

https://doi.org/10.1007/s11605-015-2918-9

6. Burt MJ, Chambers ST, Chapman BA, Strack MF, Troughton WD. Two cases of Caroli's disease: diagnosis and management. J Gastroenterol Hepatol. 1994;9(2):194-7. https://doi.org/10.1111/j.1440-1746.1994.tb01242.x

7. Ciambotti GF, Ravi J, Abrol RP, Arya V. Right-sided monolobar Caroli’s disease with intrahepatic stones: nonsurgical management with ERCP. Gastrointest Endosc. 1994;40(6):761-4. https://doi.org/10.1016/S0016-5107(94)70125-3

8. Bakhotmah MA. Successful control of external biliary fistula by using SMS 201-995 in a child. HPB Surg. 1996;9(3):183-4. https://doi.org/10.1155/1996/80945

9. Yuksel A, Has R, Isikoglu M, Suoglu O. Prenatal diagnosis of Caroli's disease. Ultrasound Obstet Gynecol. 2002; 19(5):525-6. https://doi.org/10.1046/j.1469-0705.2002.00692.x

10. Vlachogiannakos J, Potamianos S, Triantos C, Makri I, Imvrios G, Patsiaoura K, et al. Monolobar Caroli's disease complicated by cholangiocarcinoma in a 70-year-old man, previously asymptomatic. Gastrointest Endosc. 2004;60(2):297-300. https://doi.org/10.1016/s0016-5107(04)01572-x

11. Gold DM, Stark B, Pettei MJ, Levine JJ. Successful use of an internal biliary stent in Caroli's disease. Gastrointest Endosc. 1995;42(6):589-92. https://doi.org/10.1016/s0016-5107(95)70018-8

12. Marchal GJ, Desmet VJ, Proesmans WC, Moerman PL, Van Roost WW, Van Holsbeeck MT, et al. Caroli disease: high-frequency US and pathologic findings. Radiology. 1986;158(2):507-11. https://doi.org/10.1148/radiology.158.2.3510448

13. Sood GK, Mahapatra JR, Khurana A, Chaudhry V, Sarin SK, Broor SL. Caroli disease: computed tomographic diagnosis. Gastrointest Radiol. 1991;16(3):243-4. https://doi.org/10.1007/BF01887356

14. Choi BI, Yeon KM, Kim SH, Han MC. Caroli disease: central dot sign in CT. Radiology. 1990;174(1):161-3. https://doi.org/10.1148/radiology.174.1.2294544

15. Hermansen MC, Starshak RJ, Werlin SL. Caroli disease: the diagnostic approach. J Pediatr. 1979;94(6):879-82. https://doi.org/10.1016/s0022-3476(79)80206-1

16. Pavone P, Laghi A, Catalano C, Materia A, Basso N, Passariello R. Caroli's disease: evaluation with MR cholangiopancreatography (MRCP). Abdom Imaging. 1996;21(2):117-9. https://doi.org/10.1007/s002619900026

17. Pinós T, Xiol X, Herranz R, Figueras C, Catalá I. Caroli’s disease versus polycystic hepatic disease. Differential diagnosis with Tc-99m DISIDA scintigraphy. Clin Nucl Med. 1993; 18(8):664-7. https://doi.org/10.1097/00003072-199308000-00008

18. Sharma R, Mondal A, Taneja V, Rawat HS. Radionuclide scintigraphy in Caroli's disease. Indian J Pediatr.
1997;64(1):105-7.

https://doi.org/10.1007/BF02795788

19. Kaiser JA, Mall JC, Salmen BJ, Parker JJ. Diagnosis of Caroli disease by computed tomography: report of two cases. Radiology. 1979;132(3):661-4. https://doi.org/10.1148/132.3.661

20. Herman TE, Siegel MJ. Autosomal recessive polycystic disease with biliary dysgenesis. J Perinatol. 2001;21(5):339-40. https://doi.org/10.1038/sj.jp.7200224

21. Desroches J, Spahr L, Leduc F, Pomier-Layrargues G, Picard M, Picard D, Chartrand R, Morais J. Noninvasive diagnosis of Caroli syndrome associated with congenital hepatic fibrosis using hepatobiliary scintigraphy. Clin Nucl Med. 1995;20(6):512-4. https://doi.org/10.1097/00003072-199506000-00009

22. Won JH, Choi SY, Lee HK, Yi BH, Lee MH, Jung MJ. Accessory gallbladder in an intrahepatic location mimicking a cystic tumor of the liver: A case report. Medicine (Baltimore). 2016;95(44):e5293. https://doi.org/10.1097/MD.0000000000005293

23. Aguilera V, Rayón M, Pérez-Aguilar F, Berenguer J. Caroli’s syndrome and imaging: report of a case. Rev Esp Enferm Dig. 2004;96(1):74-6. https://doi.org/10.4321/s1130-01082004000100009

24. Jarry J, Leblanc F, Saric J. Maladie de Caroli monolobaire. Presse Med. 2010;39(7-8):847-8. French. https://doi.org/10.1016/j.lpm.2009.10.018

25. Lyons TJ, Benbow EW, Taylor PM, McCloy RF. Inflammatory pseudotumour of the liver: antecedent causes and clinical experience. J Hepatol. 1993;19(2):273-8. https://doi.org/10.1016/s0168-8278(05)80582-3

26. Evans-Jones G, Cudmore R. Choledochal cyst and congenital hepatic fibrosis. J Pediatr Surg. 1990;25(12):1259-60. https://doi.org/10.1016/0022-3468(90)90524-d

27. Senyüz OF, Yesildag E, Kuruoglu S, Yeker Y, Emir H. Caroli's disease in children: is it commonly misdiagnosed? Acta Paediatr. 2005;94(1):117-20. https://doi.org/10.1111/j.1651-2227.2005.tb01798.x

28. Ninan VT, Nampoory MR, Johny KV, Gupta RK, Schmidt I, Nair PM, et al. Caroli's disease of the liver in a renal transplant recipient. Nephrol Dial Transplant. 2002;17(6):1113-5. https://doi.org/10.1093/ndt/17.6.1113

29. Fulcher AS, Turner MA, Sanyal AJ. Case 38: Caroli disease and renal tubular ectasia. Radiology. 2001;220(3):720-3. https://doi.org/10.1148/radiol.2203000825

30. Esmer C, Alvarez-Mendoza A, Lieberman E, Del Castillo V, Ridaura-Sanz C. Liver fibrocystic disease and polydactyly: proposal of a new syndrome. Am J Med Genet. 2001;101(1):12-6. https://doi.org/10.1002/ajmg.1314

31. Jenss H, Krause FJ. Caroli-Syndrom-Zufallsbefund bei der postoperativen T-Drain-Cholangiographie. Rofo. 1983;139(5):576-8. https://doi.org/10.1055/s-2008-1055953 
32. Treacy PJ, Worthley CS, Harley HA. Computed tomography infusion choledochography in the management of Caroli's syndrome. Aust N Z J Surg. 1997;67(4):221-2. https://doi.org/10.1111/j.1445-2197.1997.tb01947.x

33. Zangger P, Grossholz M, Mentha G, Lemoine R, Graf JD, Terrier F. MRI findings in Caroli's disease and intrahepatic pigmented calculi. Abdom Imaging. 1995;20(4):361-4. https://doi.org/10.1007/BF00203372

34. Wu KL, Changchien CS, Kuo CM, Chuah SK, Chiu YC, Kuo CH. Caroli's disease - a report of two siblings. Eur J Gastroenterol Hepatol. 2002;14(12):1397-9. https://doi.org/10.1097/00042737-200212000-00019

35. Hernández-Ortiz J, Corona R, Méndez-Sánchez N. Caroli’s disease and choledochal cyst. Ann Hepatol. 2002;1(4):196. https://doi.org/10.1016/S1665-2681(19)32166-0

36. Chiba T, Shinozaki M, Kato S, Goto N, Fujimoto H, Kondo F. Caroli's disease: central dot sign re-examined by CT arteriography and CT during arterial portography. Eur Radiol. 2002;12(3):701-2. https://doi.org/10.1007/s003300101048

37. Cheung VT, Joshi D, Amin Z, Webster GJ. Fever and right upper quadrant pain in a 24-year-old male. Caroli disease and splenomegaly suggesting portal hypertension. Gut. 2014;63(10):1626, 1625 . https://doi.org/10.1136/gutjnl-2014-306987

38. Makino I, Tani T, Shimizu K, Takamura H, Fujimura T, Nishimura G, et al. [A case of monolobar Caroli's disease]. Nihon Shokakibyo Gakkai Zasshi. 2006;103(3):316-21.

39. Badura J, Król R, Kurek A, Hartleb M, Cierpka L. Lefthemihepatectomy as a method of treatment of locally limited Caroli disease. Pol Przegl Chir. 2013;85(11):663-5. https://doi.org/10.2478/pjs-2013-0100

40. Cha BH, Lee SH, Hwang JH, Kim SY, Kim HY. [A case of caroli disease with biliary stones]. Korean J Gastroenterol. 2009;54(4):201-4. https://doi.org/10.4166/kjg.2009.54.4.201

41. Deimel A, Sturm J, Vielfort T, Zöpf T.

Oberbauchschmerzen und Fieberschübe bei einer 44-jährigen Philippinerin. Internist (Berl). 2018;59(3):276-281. https://doi.org/10.1007/s00108-017-0320-2

42. Bodas A, Maluenda C, Rivilla F, Ortega L, Pérez O. Caroli’s syndrome and horseshoe kidney: a case report. Acta Paediatr. 2001;90(4):469-71.

43. Dhanjal NS, Sharif AW, Rosenfelder NA, Lim AK, TaylorRobinson SD. Cystic duct dilatation after cholecystectomy in fibropolycystic liver disease. J Hepatol. 2005;43(1):192. https://doi.org/10.1016/j.jhep.2004.08.027

44. Navascués R, Quiñones L, Guinea O, Guerediaga F. Enfermedad de Caroli en paciente portador de trasplante renal por poliquistosis. Nefrología. 2005;25(3):336-337.

45. Lesmana CR, Harris A, Kooshartoro A, Poerniati K, Hukom R, Czeresna HS, et al. Hepatic cirrhosis caused by Caroli disease. Acta Med Indones. 2005;37(1):33-5.

46. Bonet J, Oller B, Castellote E, Rodriguez N. Caroli’s disease and cystic kidney disease in a woman. Nephron.
1996;73(2):310-1.

https://doi.org/10.1159/000189058

47. Takatsuki M, Uemoto S, Inomata Y, Egawa H, Kiuchi T, Hayashi M, et al. Living-donor liver transplantation for Caroli's disease with intrahepatic adenocarcinoma. J Hepatobiliary Pancreat Surg. 2001;8(3):284-6. https://doi.org/10.1007/s005340170030

48. Loreno M, Bo P, Senzolo M, Cillo U, Naoumov N, Burra P. Successful pregnancy in a liver transplant recipient treated with lamivudine for de novo hepatitis B in the graft. Transpl Int. 2005; 17(11):730-4. https://doi.org/10.1007/s00147-004-0785-5

49. Braga AC, Calheno A, Rocha H, Lourenço-Gomes J. Caroli's disease with congenital hepatic fibrosis and medullary sponge kidney. J Pediatr Gastroenterol Nutr. 1994; 19(4):464-7. https://doi.org/10.1097/00005176-199411000-00018

50. Charny CK, Ling P, Botet J, Blumgart LH. Clinical observation: congenital absence of the left portal vein in a patient undergoing hepatic resection. HPB Surg. 1997;10(5):3236; discussion 326-7. https://doi.org/10.1155/1997/18965

51. Ozkurt S, Canaz F, Temiz G, Sahin G, Yalcin AU. Unusual renal manifestation of Caroli disease: AA amyloidosis. Ren Fail. 2012;34(7):930-3. https://doi.org/10.3109/0886022X.2012.690923

52. Lall NU, Hogan MJ. Caroli disease and the central dot sign. Pediatr Radiol. 2009;39(7):754. https://doi.org/10.1007/s00247-009-1147-3

53. Lefere M, Thijs M, De Hertogh G, Verslype C, Laleman W, Vanbeckevoort D, et al. Caroli disease: review of eight cases with emphasis on magnetic resonance imaging features. Eur J Gastroenterol Hepatol. 2011;23(7):578-85. https://doi.org/10.1097/MEG.0b013e3283470fcd

54. Levin G, Sabag L, Khatab AA, Rottenstreich A. An unusual case of pregnancy in a patient with Caroli's syndrome. Eur J Obstet Gynecol Reprod Biol. 2018;228:333-335. https://doi.org/10.1016/j.ejogrb.2018.06.030

55. Perricone G, Vanzulli A. Education and imaging. Hepatology: "central dot sign" of Caroli syndrome. J Gastroenterol Hepatol. 2015;30(2):234. https://doi.org/10.1111/jgh.12828

56. Takaki Y, Takahashi M, Ueno S, Fukui K. Radiologic demonstration of Caroli's disease: a case report. J Comput Tomogr. 1986;10(2):153-6. https://doi.org/10.1016/0149-936x(86)90068-8

57. Watschinger B, Schwaighofer B, Wrba F, Pohanka E, Kovarik J. Secondary hypersplenism due to Caroli syndrome complicating immunosuppression in a renal allograft recipient. Nephron. 1989;51(3):413-5. https://doi.org/10.1159/000185334

58. Saracíbar E, Martín J, Sedano E. Pericarditis constrictiva y quistes hepáticos unilobulares. Revista clínica española. 2001;201(8):483-484. https://doi.org/10.1016/S0014-2565(01)70887-9 
59. Shenoy P, Zaki SA, Shanbag P, Bhongade S. Caroli’s syndrome with autosomal recessive polycystic kidney disease. Saudi J Kidney Dis Transpl. 2014;25(4):840-3. https://doi.org/10.4103/1319-2442.135176

60. Ahmed Z, Pemira S, Chaoji, Mundle R. Caroli’s disease-a case report. Journal of Evolution of Medical and Dental Sciences. 2013;2(41):7936-7939. https://doi.org/10.14260/jemds/1398

61. Bawany MZ, Alaradi O, Nawras A. Caroli’s syndrome in a post renal transplant patient: case report and review of the literature. Saudi J Gastroenterol. 2012;18(1):59-61. https://doi.org/10.4103/1319-3767.91728

62. Kil H, Choi EY, Jeong JI, Park CS, Park SM, Kim SH, et al. A case of simple type Caroli's disease confined to right lobe of the liver. Korean J Gastroenterol. 2007;50(4):271-6.

63. Kumar A, Akselrod D, Prikis M. Caroli Disease Revisited: A Case of a Kidney Transplant Patient With Autosomal Polycystic Kidney Disease and Recurrent Episodes of Cholangitis. Transplant Proc. 2019;51(2):541-544. https://doi.org/10.1016/j.transproceed.2018.12.025

64. Carrera C, Castiella A, Fernandez J, Cosme A, GarciaBengoechea M. Caroli's disease diagnosed by magnetic resonance cholangiopancreatography. Eur J Gastroenterol Hepatol. 2002;14(5):577. https://doi.org/10.1097/00042737-200205000-00019

65. Sans M, Rimola A, Navasa M, Grande L, García-Valdecasas $\mathrm{JC}$, Andreu $\mathrm{H}$, et al. Liver transplantation in patients with Caroli's disease and recurrent cholangitis. Transpl Int. 1997;10(3):241-4. https://doi.org/10.1007/s001470050050

66. Kim RD, Book L, Haafiz A, Schwartz JJ, Sorensen JB, Gonzalez-Peralta RP. Liver transplantation in a 7-month-old girl with Caroli’s disease. J Pediatr Surg. 2011;46(8):1638-41. https://doi.org/10.1016/j.jpedsurg.2011.04.011

67. Izawa K, Tanaka K, Furui J, Matsumoto T, Segawa T, Tsunoda T, et al. Extended right lobectomy for Caroli's disease: report of a case and review of hepatectomized cases in Japan. Surg Today. 1993;23(7):649-55. https://doi.org/10.1007/BF00311917

68. Murcia S, Serrano B. Enfermedad y síndrome de Caroli. Presentación de dos casos y revisión de literatura. Rev Fac Med. 2002;50(1):88-91.

69. Sinha RJ, Sharma A, Singh V, Pandey S. Medullary sponge kidney and Caroli's disease in a patient with stricture urethra: look for the hidden in presence of the apparent. BMJ Case Rep. 2018;11(1):bcr2018226746. https://doi.org/10.1136/bcr-2018-226746

70. Martínez A, Gorriz J, Bover J, Segura-de la Morena J, Cebollada J, Escalada J, et al. Documento de consenso para la detección y manejo de la enfermedad renal crónica. Nefrología. 2014;34(2):243-62.

71. Balsells J, Margarit C, Murio E, Lazaro JL, Charco R, Vidal MT, et al. Adenocarcinoma in Caroli's disease treated by liver transplantation. HPB Surg. 1993;7(1):81-6; discussion 86-7. https://doi.org/10.1155/1993/61048

72. Higuera F, Servín A, Navarro A, Soto-Martínez K, CruzEstrada A, Alexanderson-Rosas EG. Identificación de principales factores predictivos relacionados con mortalidad hepática y muerte cardiovascular en pacientes cirróticos con diabetes tipo 2: estudio de cohorte histórica con seguimiento a 10 años. Revista de Gastroenterología de México. 2018;83( supl 2):9-13. 